\title{
PARTO SEGURO: A PERCEPÇÃO DE UMA EQUIPE DE ENFERMAGEM NO USO DO
}

\author{
CHECKLIST
}

\author{
SAFE CHILDBIRTH: THE PERCEPTION OF A NURSING STAFF IN USE OF THE
}

\author{
CHECKLIST
}

\author{
Michele Machado Cunha ${ }^{1}$ \\ Denise Maccarini Tereza ${ }^{2}$ \\ Rozilda Lopes de Souza ${ }^{3}$ \\ Cecília Marly Spiazzi dos Santos ${ }^{4}$ \\ Recebido em: 29 nov. 2017 \\ Aceito em: 18 jun. 2018
}

RESUMO: Pesquisa de abordagem qualitativa e exploratória com objetivo de conhecer a percepção da equipe de Enfermagem na aplicação do Checklist para o Parto Seguro em um hospital privado do Extremo Sul Catarinense. Aplicou-se um questionário semiestruturado com 10 colaboradores da equipe. A análise de dados foi realizada com análise de conteúdo, contendo informações sobre área de atuação atual, preenchimento e tempo utilizado do checklist do Parto Seguro, opinião sobre o checklist e o gerenciamento de riscos, contribuição do checklist na prática do trabalho, dificuldades vivenciadas no manuseio e preenchimento, comunicação da equipe durante a confirmação dos itens, as potencialidades e fragilidades na utilização do checklist do parto seguro e sugestões sobre a temática. A prática do uso do instrumento proporciona uma maior qualidade na assistência, auxiliando a equipe de enfermagem durante a checagem dos itens, prestando os cuidados necessários em todo o processo do Parto e Nascimento. É possível concluir que a percepção da equipe de enfermagem sobre o uso do checklist do parto seguro tem como centro do cuidado a parturiente e o recém-nascido e suas práticas assistenciais estão voltadas a segurança do cliente. $O$ estudo mostrou que este modelo de segurança amplia as possibilidades de parto seguro nesta instituição.

Palavras-chave: Parto. Segurança. Gestão de riscos. Checklist. Enfermagem.

ABSTRACT: This Qualitative and exploratory research in order to know the perception of nursing staff in the implementation of the Checklist for safe delivery in a private hospital in the far South of Santa Catarina. Applied a semi-structured questionnaire with 10 employees of the team. Data analysis was performed using content analysis, containing information on current operating area, and fill time used the checklist of safe delivery, opinion about the checklist and the risk management, the contribution checklist in the practice of work, difficulties experienced in handling and filling, communication from the team during the confirmation of the items, the potentialities

\footnotetext{
1 Enfermeira. UNESC - Universidade do Extremo Sul Catarinense - Criciúma - SC - Brasil. Endereço para correspondência: Rua Ana Herculano Magalhães, 18. Renascer. Criciúma - SC. CEP: 88.8016-015. E-mail: enfmichelemachado@gmail.com.

2 Enfermeira. Mestre em Ciências da Saúde - UNESC - Universidade do Extremo Sul Catarinense - Criciúma - SC - Brasil. E-mail: denisemaccarini@yahoo.com.br.

${ }^{3}$ Enfermeira. Mestre em Ciências da Saúde - UNESC - Universidade do Extremo Sul Catarinense - Criciúma - SC - Brasil. E-mail: rozildalopes@unesc.net.

${ }^{4}$ Enfermeira. Mestre em Ciências da saúde - UNESC - Universidade do Extremo Sul Catarinense -Criciúma SC - Brasil. E-mail: marly@unesc.net.
} 
and weaknesses in the use of the checklist of safe delivery and suggestions on the subject. The practice of the use of the instrument provides a higher quality in assistance, assisting the nursing staff during the checking of the items, providing the necessary care in the entire process of childbirth and Birth. It can be concluded that the perception of nursing staff on the use of the checklist of the safe delivery of care center has the mother and the newborn and their welfare practices are geared to client security. The study showed that this security model extends the possibilities for safe delivery in this institution.

Keywords: Childbirth. Security. Risk management. Checklist. Nursing.

\section{INTRODUÇÃO}

A Lista de Verificação da OMS para Partos Seguros foi desenvolvida como um instrumento para melhorar a qualidade dos cuidados prestados no parto e nascimento, é uma lista organizada de práticas essenciais baseada em evidencias, que visa às principais causas de morte materna por causas intraparto e das mortes neonatais em todo o mundo. Cada item da Lista de Verificação é uma ação crítica que, se não for cumprida, poderá provocar sérios danos a mãe, ao recém-nascido ou a ambos (OMS, 2017).

Com o uso do checklist pode-se aprimorar o gerenciamento de risco nas instituições, com foco na segurança dos pacientes melhorando a qualidade da assistência prestada, acredita-se que o gerenciamento de risco possibilita aos profissionais de enfermagem avaliar o cuidado oferecido observando e propondo melhores práticas que minimizem e evitam os eventos adversos (GOMES et al., 2016a).

A prática do uso do instrumento proporciona uma maior qualidade dos partos realizados em unidades de saúde para as mães e os cuidados essenciais aos recémnascidos, auxiliando aos profissionais responsáveis na checagem dos itens para prestar os cuidados necessários em todo o processo do Parto e Nascimento. É importante a aprovação dos profissionais envolvidos na utilização do checklist, para assegurar o uso eficaz da Lista de Verificação. E especialmente o conhecimento dos diferentes níveis administrativos do setor, para assegurar que conhecem o processo da Lista de Verificação, o que é a lista, como funciona e porque o preenchimento de cada item é tão importante (OMS, 2017).

Diante destas reflexões motivou se a realização da pesquisa para analisar a percepção dos profissionais sobre o checklist do Parto Seguro, que segundo a OMS (2017), o instrumento vem para melhorar a segurança e a qualidade do parto e nascimento em todo o mundo. Sendo importante analisar a percepção da equipe de enfermagem para evidenciar o uso eficaz, conhecimento, suas visões críticas e avaliativas dos processos que abrangem o uso do checklist do Parto Seguro.

Considera-se que o uso do checklist do Parto Seguro garante qualidade nos registros permitindo a análise de todo processo que está sendo realizado na prestação da assistência de enfermagem; proporcionando melhoria na comunicação da equipe de 
enfermagem e soluções para possíveis problemas e um melhor atendimento.

Com intuito de conhecer a percepção entre os colaboradores participantes, tem-se como objetivo conhecer a Percepção da equipe de Enfermagem sobre o uso do Checklist do Parto Seguro.

\section{MATERIAL E MÉTODOS}

Pesquisa qualitativa, descritiva exploratória. $O$ estudo foi realizado em um Hospital privado do Extremo Sul Catarinense com 10 colaboradores da equipe de enfermagem, sendo 9 técnicas de enfermagem e 1 enfermeira atuantes na Clinica da Mulher de diferentes turnos.

Como critério de inclusão utilizou-se: ser enfermeiro ou técnico de enfermagem; estar atuando a pelo menos 3 meses na instituição; aceitar participar por meio da assinatura do TCLE; atuar em qualquer turno de trabalho. Como Critério de exclusão utilizou-se: ser menor de 18 anos; pertencer a outro setor do hospital; estar afastado por motivo de doença ou algum tipo de licença durante o período da pesquisa.

A coleta de dados foi efetuada por meio de questionário semiestruturado, aplicado pela própria pesquisadora no contexto do estudo.

A análise e interpretação dos dados foram realizadas pela análise de conteúdo, a partir da categorização dos dados, através da ordenação, classificação e análise final dos dados pesquisados.

Conforme Minayo (2009) as categorias são empregadas para estabelecer classificações, significa agrupar elementos, ideias ou expressões em torno de um conceito capaz de abranger tudo que pode ser utilizado em qualquer tipo de análise em pesquisa qualitativa.

Este trabalho foi submetido ao Comitê de ética em Pesquisa com Seres Humanos da Universidade do Extremo Sul Catarinense sendo aprovado pelo no 2.201.270/ 2017. A coleta de dados somente foi realizada após aprovação pelo referido comitê, estando de acordo com a resolução 510/2016, que regulamentam a pesquisa com humanos.

Para preservar o sigilo decorrente do questionário, de acordo com as diretrizes e normas regulamentadoras que envolvem pesquisa com Seres Humanos e Grupos Vulneráveis, utilizou-se a letra 'P' para os profissionais da equipe de enfermagem; seguido do respectivo número - $\mathrm{P} 1$ a $\mathrm{P} 10$. 


\section{RESULTADOS E DISCUSSÃO}

\section{CARACTERIZAÇÃO DO PERFIL DOS PROFISSIONAIS}

Em relação ao perfil dos profissionais entrevistados, todos são do sexo feminino; a faixa etária estabeleceu-se entre 20 a 38 anos; categoria profissional P1; P2; P4; P5; P6; P7; P8; P9 e P10 são técnicas de Enfermagem, P3 Enfermeira. O tempo de atuação dos profissionais no setor Clínica da Mulher variou de menos de 1 ano a mais de 5 anos. $O$ turno de trabalho matutino, vespertino e integral.

\section{PREENCHIMENTO E O TEMPO UTILIZADO DO CHECKLIST DO PARTO SEGURO}

Todos os profissionais atuantes da clínica da mulher preenchem o instrumento checklist do parto seguro totalizando 10 colaboradores. O tempo utilizado para 0 preenchimento do checklist é cerca de 5 a 10 min.

Segundo a OMS (2017) o checklist do Parto Seguro pode ser normalmente usado de duas maneiras: em Ler-Fazer, deve ler-se, primeiro, o item da Lista do checklist e depois preencher o item. Em Fazer-Confirmar, deve-se realizar o item solicitado e depois ler o ponto da Lista do checklist, para confirmar que a tarefa foi realizada. Pode realizar qualquer um dos métodos, embora Ler-Fazer é um dos métodos mais seguro da forma correta de realizar a checagem, especialmente ao colaborador que esteja iniciando o uso do Checklist.

\section{OPINIÃO SOBRE O CHECKLIST E O GERENCIAMENTO DE RISCOS}

A opinião dos colaboradores denotou a importância do checklist e o gerenciamento de riscos para a segurança do cliente; com identificação correta do cliente; evitando trocas, riscos e buscando a integralidade da assistência.

O checklist é considerado uma ferramenta essencial para a promoção da segurança do recém-nascido, parturiente e da própria equipe para a continuidade da assistência, conforme os relatos:

P3- "Checklist é uma ferramenta importante na segurança do paciente, enfim toda equipe envolvida no cuidado centrado da paciente [...]".

P4- "Sobre o checklist posso dizer que é uma ferramenta muito importante tanto para a segurança da mãe/bebê como para a continuidade da assistência, pois nela está todos os dados da cliente e alertas de risco."

P7- "É uma segurança para a equipe, que atenderá este paciente/ cliente, um atendimento individualizado, um cuidado individualizado com os pacientes, clientes em 
tratamento".

A promoção da assistência materna e neonatal segura é de fundamental importância, tendo em vista o grande número de clientes envolvidos e o potencial de risco de eventos adversos que podem ocorrer no processo assistencial. No Brasil, aproximadamente $98 \%$ dos partos acontecem em estabelecimentos hospitalares públicos ou privados, com aproximadamente 3 milhões de nascimentos a cada ano, totalizando, nesses segmentos, quase 6 milhões de clientes entre parturientes e recém-nascido. (BRASIL, 2014).

P8 - "Com o fluxo de pacientes, para não haver troca de pacientes, e informações de alergias e doenças que o paciente possa ter."

Em pesquisa de revisão Gomes et al. (2016b) sobre erros na administração de medicamentos encontrou como fatores desencadeadores dos erros: Troca de pacientes, erro de dosagem via errada, além de erro documental, omissão de justificativas quando necessário e outras. O erro na administração de medicamentos é responsável por deixar sequelas irreparáveis nos clientes ou até levar à morte.

P10 - "É importante para não haver troca de clientes e para ver se tem alergias, medicações etc."

É imprescindível que os profissionais de saúde busquem a qualificação e atualização periodicamente, a fim de minimizar os eventos adversos na administração de medicamentos, buscando a qualificação do cuidado. Enfatiza se a importância da identificação correta do cliente são medidas usadas para garantir a segurança do mesmo. (GOMES et al., 2016b).

P3 - "Na realização podemos identificar os riscos, assim evitar os danos e posteriormente evitar eventos adversos [...]".

P2 - "Com esses instrumentos de trabalhos tão importantes para organização da equipe além de minimizar os riscos e incertezas sobre qualquer eventualidade que possamos identificar antes das cirurgias".

Com as ações de gerenciamento de risco nas instituições de saúde, pode se observar por meio das falas a melhoria na qualidade assistencial, buscam a segurança dos clientes, identificando as situações que os colocam em risco, agindo na sua prevenção e controle. Possibilitando a equipe de enfermagem avaliar o cuidado oferecido ao cliente, observando e propondo a melhoria nas práticas que minimizam os riscos. $\mathrm{O}$ uso do checklist tem a finalidade essencial de identificar os riscos precocemente, evitando danos e eventos adversos tanto com o recém-nascido como com a parturiente:

P9 - "Importante para saber informações do paciente que vão ser utilizados medidas para a continuidade ao tratamento. Por exemplo, gestante com diabetes/ hipertensão logo após a sala de recuperação vão ser tomado medidas especiais para essa paciente que corre risco ou se é alérgica a alguma medicação."

P1 - "É importante o checklist, pois importância que vão da admissão da mãe até o 
momento da alta e devemos prestar atenção no nome, sobrenome, nome da mãe e pai. Ver se tem alergia, pré-natal, consulta".

A identificação do cliente para Tase e Tronchin (2015) é uma área de alta prioridade dentre os gerenciamentos de riscos e assistenciais nos serviços de saúde, pois, quando ocorre algum erro ou evento adverso, relativo a não conformidade na identificação, os desfechos, na maioria das situações, são preocupantes. Por outro lado, é uma prática de medidas evitáveis quando valorizada pelos profissionais de saúde. Sendo assim, apontada como uma das soluções e um componente essencial e decisivo na assistência segura, que se realizada corretamente, será passível de prevenir inúmeros erros ou eventos adversos nos múltiplos âmbitos da prática do cuidado.

P5 - "Há uma grande importância no preenchimento de dados específicos antes de qualquer procedimento realizado com o paciente evitando erros com o mesmo".

Para o profissional P6 o checklist pode possibilitar uma melhor integralidade na assistência materna e neonatal, com a humanização da assistência individualizada e humanizada, pois nesse momento vivencia fortes emoções, tanto para as parturientes como para seus familiares, porque as experiências vivenciadas ficaram registradas de uma forma positivas ou negativas, para o resto de suas vidas. (BRASIL, 2014b).

P6 - "Sim bom para o paciente, cuidado físico, psíquico do mesmo”.

Portanto, a preocupação com a segurança deve ter um olha integral incluindo aspectos emocionais, culturais e sociais envolvidos neste momento único para as Parturientes e seus familiares (BRASIL, 2014b).

O Programa Nacional de Segurança do Paciente (PNSP), lançado pelo Ministério da Saúde (MS), por meio da publicação da Portaria $n^{\circ}$. 529, de 1 de abril de 2013. Entendese que:

\begin{abstract}
Considerando que a gestão de riscos voltada para a qualidade e segurança do paciente englobam princípios e diretrizes, tais como a criação de cultura de segurança; a execução sistemática e estruturada dos processos de gerenciamento de risco; a integração com todos os processos de cuidado e articulação com os processos organizacionais do serviço de saúde; as melhores evidências disponíveis; a transparência, a inclusão, a responsabilização e a sensibilização e capacidade de reagir a mudanças; e Considerando a necessidade de se desenvolver estratégias, produtos e ações direcionadas aos gestores, profissionais e usuários da saúde sobre segurança do paciente, que possibilitem a promoção da mitigação da ocorrência de evento adverso na atenção à saúde. (BRASIL, 2013, p.1).
\end{abstract}

Os resultados evidenciaram uma grande importância na segurança do cliente com o objetivo de minimizar possíveis erros e incertezas, contribuindo assim para a organização do processo na busca da qualidade da integralidade na assistência prestada a parturiente e ao recém-nascido. 
As contribuições do checklist na prática de trabalho da equipe de enfermagem possibilita a segurança do paciente, comunicação da equipe para as certezas do processo sendo fundamental na qualidade do cuidado conforme estudos já realizados, o uso do checklist não atrapalha a assistência prestada.

O checklist na prática de trabalho possibilita a segurança do paciente e equipe, através da organização dos procedimentos que necessitam ser prestados tornando se uma prática essencial segundo os relatos dos colaboradores:

P1 - "Sim Pois é a segurança do paciente".

P8 - "Para a segurança do paciente e equipe".

P9 - "Sim Reforça a segurança para nós, para o paciente".

P10 - "Para dar segurança para o cliente, médico e para a enfermagem".

P3 - "Muito com o objetivo na segurança do paciente".

No dizer de Neves et al. (2014) a proteção no ambiente de trabalho não está individualizada apenas na proteção do colaborador envolvido com o procedimento, mas sim de toda a equipe na prática assistencial proporcionando um cuidado integral do colaborador e do cliente. O uso de instrumentos que ajudam no cotidiano dos colaboradores fornece segurança para terem a certeza que estão prestando um cuidado de qualidade embasado em evidencias cientificas.

Destaca-se no relato do colaborador P4 que o checklist contribui para a identificação das certezas na prática de trabalho: P4 - "O uso do instrumento contribui para termos as certezas que estamos seguindo um processo correto. Para termos nosso laudo certo".

Perante as falas dos colaboradores sobre o checklist como um instrumento que visa também à segurança do Profissional da saúde a NR 26 estabelece diretrizes básicas para a implementação de medidas de proteção à segurança e à saúde dos trabalhadores na área da saúde:

Esta Norma Regulamentadora - NR tem por finalidade estabelecer as diretrizes básicas
para a implementação de medidas de proteção à segurança e à saúde dos trabalhadores
dos serviços de saúde, bem como daqueles que exercem atividades de promoção e
assistência à saúde em geral. Para fins de aplicação desta NR entende-se por serviços de
saúde qualquer edificação destinada à prestação de assistência à saúde da populaça, e
todas as ações de promoção, recuperação, assistência, pesquisa e ensino em saúde em
qualquer nível de complexidade. (BRASIL, 2005, p.1).

A qualidade da assistência atende as necessidades dos clientes e tem o intuito de promover serviços de excelência envolvendo toda a equipe.

Além das certezas o checklist é fundamental para proporcionar qualidade e segurança no parto: (P2) "Ferramenta fundamental para melhorar a qualidade dos cuidados 
dispensados as mulheres que dão à luz e recém-nascidos".

Broca e Ferreira (2015) destacam que a enfermagem é uma profissão praticada em equipe, sendo uma extensão continuada dos envolvidos na assistência. Valorizando e enfrentando, os desafios da comunicação entre relações durante o cuidado, sendo um agente transformador na equipe multiprofissional para uma comunicação sensível.

A comunicação da equipe é fator essencial para a aplicação correta do checklist perante os seguintes relatos:

P5 - "Sim. Com toda a equipe que receber este paciente estará ciente dos danos e riscos que podem ocorrer durante o procedimento".

P7 - "Sim. Passa as informações referentes ao paciente cliente e o cuidado que devo oferecer ao paciente cliente".

A comunicação entre a equipe é fundamental para um bom desenvolvimento do checklist, essa relação qualifica o relacionamento da equipe multiprofissional onde todos participam com a finalidade de prestar uma assistência qualificada, compartilhando as informações entre as equipes de diferentes turnos e o conhecimento da história da parturiente por profissionais que não acompanharam a sua gestação.

P3 - "[...] a ferramenta é valiosa também para os médicos onde em algumas situações o profissional não conhecia a história do paciente e o plantão realiza o procedimento e com a comunicação efetiva e sua realização torna-se o uso do instrumento a prática essencial".

A comunicação apresenta se em constante mudança e as formas de se comunicar qualificam o relacionamento da equipe e contribuem com elementos para a estruturação da equipe influenciando a efetividade do processo. (BROCA; FERREIRA, 2015).

Segundo a colaboradora P6 o checklist é uma fonte de informação que pode ocasionar burocracia e pouco contato direto com o cliente:

P6 - "Sim, pois é fonte de informação. Não porque dependendo do procedimento acaba sendo muito papel e pouco contato com o cliente".

Todos os itens da Lista de Verificação são importantes como fonte de informação para todos os partos, embora possa parecer que seu preenchimento seja moroso, logo permitirá ao colaborador executar a prática da assistência com maior segurança e facilidade em seguir o processo necessário e tão importante durante a realização do parto. (OMS, 2017).

\section{DIFICULDADES VIVENCIADAS NO MANUSEIO E PREENCHIMENTO DO CHECKLIST}

As dificuldades vivenciadas que envolvem o manuseio e preenchimento do checklist, segundo a equipe de Enfermagem, relacionam-se principalmente com as informações incompletas do cartão do pré-natal; falta de comunicação e ainda a 
organização do tempo da equipe multiprofissional durante a realização do checklist; além da falta de conhecimento das parturientes sobre informações da gestação.

É imprescindível a realização do pré-natal para identificar situações de riscos e intercorrências no clico gravídico que possa interferir no curso normal, possibilitando um diagnostico precocemente. O cartão da gestante deve estar preenchido corretamente com todas as informações necessárias como a identificação da gestante, as consultas realizadas, calendário de vacina e exames solicitados.

P5 - "Cartão pré-natal incompleto".

P6 - "Existe a dificuldade quando o cartão pré-natal da paciente está incompleto ou inelegível".

P7 - "O não preenchimento completo".

A atenção ao pré-natal segundo Carpes, Ressel e Stumm (2016) consiste na prevenção, promoção da saúde e tratamento de problemas que ocorrem durante 0 processo gestacional interferindo no parto e pós-parto, implicando na dificuldade da assistência quanto à falta de conhecimento da gestante em repassar informações importantes para sua saúde e do recém-nascido.

Assim durante o pré-natal é o momento oportuno para realizar educação em saúde incentivando o empoderamento da gestante na busca do conhecimento para seu cuidado e do recém-nascido. Conforme relato da P2: "Quando o paciente não sabe dar as informações completas, exemplo doenças infectocontagiosas, dosagem de medicações em uso".

Para otimizar o cuidado com o uso do checklist, a equipe de enfermagem depende da colaboração dos demais integrantes da equipe multidisciplinar, uma boa comunicação é peça chave para que profissionais possam trabalhar em um ambiente de ajuda mútua. Compartilhando conhecimentos, responsabilidades, trocando informações e propondo, sugerindo e modificando ações, resultando em um verdadeiro trabalho em equipe. Possibilitando assim um melhor enfrentamento das situações adversas nos relacionamentos interpessoais, permitindo desenvolver os conhecimentos, as aptidões e a confiança de que precisam para participar plenamente dessa parceria. (WALDOW, 2014).

P1 - "É importante, pois não é ouvido por todos os participantes."

P9 - "Às vezes a cirurgia, o procedimento em si é muito rápido, e o checklist é algo que precisa ser feito com calma e clareza e muitas vezes o próprio médico não quer ouvir, somos orientados a falar mesmo assim".

Segundo a fala do colaborador P4, a falta de atenção e o não conhecimento do checklist, dificulta a assistência, esta falha é suprida por constantes treinamentos realizados na instituição para a qualidade da assistência: (P4) "Muitas vezes na correria do processo a falta de atenção e a falta de conhecimento da ferramenta deixamos a desejar. Mas sempre estamos em treinamento para melhor atender". 
É fundamental que o profissional tenha conhecimento e esteja treinado para a utilização do checklist, estando apto a interromper qualquer das etapas, caso julgue necessário, ou dar prosseguimento para a próxima fase. Os colaboradores envolvidos no processo necessitam ter conhecimento sobre esse instrumento, compreender a sua importância e incorporá-la na prática diária. Cada participante da equipe é peça chave para o sucesso da ação, considerando que todos são responsáveis pela segurança do paciente. (MONTEIRO, 2014).

P3 - "São o preenchimento de todas as etapas que ele contém em algumas situações com a "pressa", a equipe acaba deixando de preencher por completo".

Perante a fala dos colaboradores P8 e P10 não relataram dificuldades no manuseio e preenchimento do checklist: P8 - "nenhuma", P10 - "nenhuma".

A aplicabilidade do checklist depende das informações contida em de todas as etapas, com conhecimento e compreendendo sua importância para a parturiente e recémnascido tanto quanto para a equipe multiprofissional na organização da assistência prestada com qualidade.

\section{COMUNICAÇÃO DA EQUIPE DURANTE A CONFIRMAÇÃO DOS ITENS}

A comunicação da equipe durante a confirmação dos itens acontece antes do procedimento sendo importante a participação de todos, mesmo quando alguns profissionais da equipe multidisciplinar ignoram ou não gostam de realizar em voz alta a confirmação dos itens, assim nem sempre acontecendo a confirmação dos itens de cada etapa do checklist.

O envolvimento multidisciplinar na realização de um processo de segurança é fundamental, pois contribui para a receptividade e adesão ao processo. A interação no ambiente influencia no cuidado da parturiente e recém-nascido. Constata as seguintes falas:

P1 - "Sim. Antes o procedimento é realizado o checklist em sala cirúrgica".

P2 - "Sim. Sempre importante realizar o checklist em voz alta em sala de cirurgia certificando se que todos envolvidos participem das informações ali anotadas".

P4 - "Sim. Confirmamos todos os dados antes da indução anestésica".

Levando em consideração que o trabalho em equipe é inerente à enfermagem, assim como afirma Copelli et al. (2015) demonstra que a relação interpessoal entre os integrantes da equipe multiprofissional beneficia o desenvolvimento do enfermeiro gestor, uma vez que são estabelecidas relação de confiança e ajuda mútua no ambiente de trabalho, contribuindo para a maior satisfação do profissional com o trabalho e qualidade do cuidado oferecido. Para que o trabalho em equipe ocorra de forma coesa, faz-se necessário que os profissionais estejam preparados para agir com competência: 
P3 - "Sim. Procuramos sempre induzir a equipe na participação, realizamos com o profissional medico em tempo real, ocorre em alguns momentos o esquecimento de realizar a etapa, assim nós gestores chamamos a equipe para uma reflexão importante do preenchimento da ferramenta".

A participação no cuidado prestado sob o olhar da integralidade promovida pela educação permanente permite a ampliação e a preocupação da dimensão do cuidado e da segurança, além de fortalecer a responsabilização dos colaboradores sendo transformação da prática e da tomada de decisão em direção a sua proteção e do cliente. (BROCA; FERREIRA, 2015).

P5 - "Não, alguns médicos ignoram e outros têm uma boa aceitação."

P6 - "Depende de qual cirurgião, sendo que algum não 'gosta' que realize o checklist em voz alta".

P7 - "Muitas vezes o próprio médico para médico, realiza a confirmação deixando a equipe de lado".

A resistência de alguns profissionais da equipe multidisciplinar também foi apontada como causas intervenientes no trabalho da equipe, uma vez que, para se alcançar a tão almejada assistência integral, é necessário desenvolver uma assistência interdisciplinar, e não atuar de forma individualizada ou que comprometa a qualidade do cuidado à parturiente. (DODOU et al., 2017).

P9 - "É para ser, porém nem sempre, pois às vezes o médico chega atrasado, a colega de trabalho (circulante) tem que sair da sala para fazer outro procedimento".

P10 - "Sim. Mas nem sempre".

A comunicação deve estar presente em todas as etapas de um procedimento, sendo das informações adquiridas à formação do planejamento para a assistência que será prestada. A equipe depende de um envolvimento integral de todos os participantes para juntos desenvolverem as ações para o cuidado oferecido.

\section{AS POTENCIALIDADES E FRAGILIDADES NA UTILIZAÇÃO DO CHECKLIST DO}

\section{PARTO SEGURO}

A utilização do checklist apresenta como potencialidades, segundo as participantes do estudo, a humanização do parto prestando um atendimento individualizado e seguro para a parturiente e recém-nascido evitando possíveis riscos adversos com o uso do instrumento. Dentre as fragilidades com a utilização do checklist estão a falta de dados e o não preenchimento correto, a falta de comunicação da equipe multidisciplinar e a parturiente.

Na prática o checklist contribui para o processo de trabalho, sendo um facilitador nas rotinas que devem ser seguidas pela equipe multiprofissional. As potencialidades do 
checklist estão relacionados à qualidade das práticas assistenciais na segurança da parturiente e recém-nascido como percebe se nas falas a seguir:

(P1) "É a humanização do parto em gestantes de baixo risco obstétrico um parto seguro enfatizam parto vaginal, nos incentivos econômicos na utilização de tecnologia”.

A melhoria de qualidade na assistência ao parto e ao nascimento no Brasil tem incentivado os profissionais de saúde a implementar ações de humanização provocando o estímulo de novas posturas frente ao parto e nascimento. (MARIA et al., 2017).

P7 - "Potencial, dar um atendimento adequado individualizado seguro para 'paciente' cliente".

O uso do checklist na preparação da parturiente para realização do procedimento que envolve o pré-operatório tem como uma das principais finalidades a conferência das informações obtidas sobre sua identificação e sobre adequação da montagem da sala para o procedimento proposto. Os riscos a parturiente e ao recém-nascido são uma realidade presente e cabe à equipe multiprofissional no processo propor estratégias e estabelecer barreiras para garantir a segurança. (ROSCANI, 2017).

P2 - "Tem o potencial de garantir a segurança do paciente no pré-operatório".

P4 - "Temos todos os questionamentos para evitar risco no procedimento".

P5 - "Contribui para segurança do paciente".

Logo, compreende se que as fragilidades da utilização do checklist do parto seguro tendem a influenciar no resultado final, que é assistência prestada. Esta, por sua vez, deve estar pautada na excelência do cuidado que envolve desde a forma correta de manusear do instrumento até a forma técnica, científica e humanizada do parto. (DODOU et al., 2017).

P2 - "[...] a fragilidade ocorre quando não identificamos possíveis eventos quanto para o paciente, quanto para a equipe envolvida".

P3 - "Falta de preenchimento de etapa furamento (incompleto)".

De acordo com P4 a fragilidade vivenciada no uso do checklist quando: "São sobre dados que coletamos do RN, onde temos que entrar em contato com o berçário, às vezes passa despercebido".

P5 - "A fragilidade quando o paciente não relata dados."

P7 - "As fragilidades, quando não é realizado corretamente".

O trabalho da equipe multidisciplinar é um elo voltado ao cuidado prestada com excelência a parturiente e ao recém-nascido, sendo importante reconhecer o trabalho do outro e considerá-lo, proporcionado através do processo de comunicação, um rompimento das barreiras que impedem ou prejudiquem as relações estabelecidas entre os profissionais de diferentes categorias, hierarquias, poder e status. Desse modo, é importante estreitar laços de comunicação efetivo, sem imposições, ruídos ou barreiras entre a equipe multidisciplinar para evitar que haja conflitos e contradições nas informações 
compartilhadas sobre a assistência prestada, evitando qualquer ação negativa no cuidado de enfermagem que por ventura venha a causar algum tipo de risco na assistência e, nesse sentido, a interação faz toda a diferença. (BROCA; FERREIRA, 2015).

P8 - "A falta de comunicação de Paciente equipe de enfermagem e médica".

P9 - "Às vezes falta de comunicação da equipe e com a paciente para esclarecer certas perguntas sobre o checklist".

P10 - "Falta de comunicação nem todas relatam realmente o que é como as doenças".

Nogueira e Rodrigues (2015) em pesquisa sobre comunicação efetiva no trabalho em equipe em saúde: desafio para a segurança do paciente consideram que o trabalho em equipe é complexo e é considerado impulsionador de transformações, sendo a comunicação efetiva ponto-chave. Para uma cultura organizacional positiva, esse aspecto representa constante desafio, e requer avaliação permanente, em que pesem as experiências vivenciadas e compartilhadas. Consideram a relevância da temática enfoco no campo da saúde e enfermagem contemporânea, associado à vivência prática em situações que são causadas por falhas no trabalho em equipe e na comunicação entre os profissionais multidisciplinar bem como a relação com o cliente, para a qualidade dos cuidados em saúde e a segurança do cliente.

\section{SUGESTÕES SOBRE A TEMÁTICA}

O tema sobre Checklist do Parto seguro é considerado um instrumento útil e importante na organização das tarefas essenciais para ofertar qualidade e segurança, sendo que os participantes ofereceram sugestões como torna-lo mais práticos, que os profissionais deem mais importância ao uso do instrumento; realizar uma comunicação efetiva com a equipe e cliente, o contato real com o paciente tendo respeito a cada opinião:

P1 - "Foi um tema muito interessante, devemos sim ter respeito a cada opinião de uma gestante, devemos respeitar o que ela opte no seu parto, mas com o checcklist seguro e um pré-natal e seu obstetra".

P9 - "O checklist vendo em si para ser uma coisa fácil e de grande importância, porem muitas vezes pela correria do dia a dia, acaba sendo difícil. Talvez se tivesse uma coisa para torná-lo mais prático e mais seguro, e ou os próprios funcionários sendo médicos também dar mais importância para ele".

Vivências no cotidiano profissional, assim como a literatura onde destacam Nogueira e Rodrigues (2015), têm indicado que a comunicação ineficaz repercute em cuidado inseguro. Ao pensar sobre comunicação no trabalho de equipes interdisciplinares em saúde, evidenciou-se que programas de treinamentos de habilidades de comunicação, simulações práticas e maneiras padronizadas para apresentar informações do cliente, estabelecem formas efetivas para transpor barreiras à comunicação ineficaz. Assim, uma 
cultura organizacional com missão embasada em objetivos e estratégias com efetivas configurações de comunicação, refletirá positivamente nos processos assistenciais e, consequentemente, na qualidade dos serviços e segurança do cliente.

P7 - 'Precisamos realizar estas comunicações coerentes, para todos 'pacientes' clientes, para toda equipe, para cada vez mais ter certeza e controle no atendimento adequado para cada 'paciente' cliente".

As sugestões ajudam continuamente nas práticas de trabalhando esclarecendo e indicando possíveis melhorias com os colaboradores que utilizam este instrumento diariamente. A partir das sugestões possibilita o encorajando para pensar novas práticas de aperfeiçoamento na assistência do parto seguro. (OMS, 2017).

\section{CONSIDERAÇÕES FINAIS}

A prática do uso do instrumento proporciona uma maior qualidade na assistência, auxiliando os profissionais multidisciplinar responsáveis durante a checagem dos itens, prestando os cuidados necessários em todo o processo de Parto e nascimento.

Evidenciou se a partir desta pesquisa que a percepção da equipe de enfermagem sobre o uso do checklist do parto seguro tem como centro do cuidado a parturiente e 0 recém-nascido e suas práticas assistenciais estão voltadas a segurança do cliente.

Os Resultados da da pesquisa denotam que a equipe de enfermagem realiza a aplicação do checklist do Parto Seguro garantindo a qualidade nos registros com uso do instrumento corretamente onde permite a análise de todo processo que está sendo realizado na assistência prestada pela equipe de enfermagem. A realização do checklist do Parto Seguro proporciona melhoria na comunicação da equipe e um melhor atendimento.

Nesta perspectiva considera-se que a percepção da equipe de enfermagem sobre o uso do Checklist do Parto Seguro, reconhece a aplicabilidade do instrumento bem como o conhecimento sobre a segurança do parto. Identificando as potencialidades e as fragilidades presentes no uso do instrumento relacionados a necessidade de garantir a segurança das práticas assistenciais. A comunicação e participação ativa da equipe multiprofissional aparecem como meio importante para investimento nas relações e para a realização do checklist, em contrapartida a resistência por parte de alguns colaboradores da equipe multiprofissional.

Este modelo de segurança para o parto seguro, amplia as possibilidades de parto seguro nesta instituição.

\section{REFERÊNCIAS}

BRASIL. Ministério do Trabalho. Segurança e saúde no trabalho em serviços de saúde. 2005. Disponível em: <http://trabalho.gov.br>. Acesso em: 31 out. 2017. 
BRASIL. Agência Nacional de Vigilância Sanitária - ANVISA. Portaria nำ 529, de 1ำ de abril de 2013. Institui o Programa Nacional de Segurança do Paciente (PNSP).

BRASIL. Ministério da Saúde. Documento de referência para o Programa Nacional de Segurança do Paciente. Fundação Oswaldo Cruz, Agência Nacional de Vigilância Sanitária. Brasília: Ministério da Saúde, 2014.

BROCA, Priscilla Valladares; FERREIRA, Márcia de Assunção. Processo de comunicação na equipe de enfermagem fundamentado no diálogo entre Berlo e King. Escola Anna Nery Revista de Enfermagem, v.19, n.3, p.467-474, 2015.

CARPES, Fabiano Fraga; RESSEL, Lucia Beatriz; STUMM, Karine Eliel. Assistência prénatal sob a ótica de gestantes e familiares. Saúde Santa Maria, Santa Maria, v.42, n.2, p.41-48, jun., 2016. Disponível em: <https://periodicos.ufsm.br>. Acesso em: 29 nov., 2017

COPELLI, Fernanda Hannah da Silva et al. Compreendendo a governança da prática de enfermagem em um centro obstétrico. Escola Anna Nery Revista de Enfermagem, v.19, n.2, 2015. Disponível em: <http//scielo.br>. Acesso em: 29 out. 2017.

DODOU, Hilana Dayana et al. Sala de parto: condições de trabalho e humanização da assistência. Cadernos Saúde Coletiva, [s.I.], v.25, n.3, p.332-338, 9 out., 2017. Disponível em: <http://scielo.br>. Acesso em: 29 out. 2017.

GOMES, Cátia Denise Perez Pereira et al. Percepção de uma equipe de enfermagem sobre a utilização do checklist cirúrgico. Sobecc. São Paulo, p.140-145, 22 jul., $2016 a$.

GOMES, Andréa Tayse de Lima et al. Erros na administração de medicamentos: evidencias e implicações na segurança do paciente. Cogitare Enferm. Parana, v.21, n.3, p.01-11, set., 2016b.

MARIA, Fernanda Lidorio et al. O significado do parto humanizado para os profissionais e as gestantes do inanna. In: JERONIMO, Rosa Nadir Teixeira (Org.). Parto humanizado: o nascimento em ambiente amortizado. Criciúma, 2017.

MONTEIRO, Fátima; SILVA, Luciana Rodrigues. "Checklist" Lista de Verificação de Segurança Cirúrgica: avaliação e intervenção. Revista de Ciências Médicas e Biológicas, v.12, n.4, p.482-485, 2014.

MINAYO, Maria Cecilia de Souza. O desafio do conhecimento. São Paulo: Hucitec, 2008.

NEVES, Heliny Carneiro Cunha et al. A segurança dos profissionais da área da saúde na perspectiva da integralidade: uma reflexão teórica. Ciência, Cuidado e Saúde. Maringá, v.13, n.4, p.770-775, dez., 2014.

NOGUEIRA, Jane Walkiria da Silva; RODRIGUES, Maria Cristina Soares. Comunicação efetiva no trabalho em equipe em saúde: desafio para a segurança do paciente. Cogitare Enfermagem. Curitiba, v.20, n.3, p.636-640, jul., 2015. Disponível em:

<http://revistas.ufpr.br>. Acesso em: 01 nov. 2017.

OMS. Organização Mundial da Saúde. Guia de Implementação da Lista de Verificação 
da OMS para Partos Seguros: melhorar a qualidade dos partos realizados em unidades de saúde para as mães e os recém-nascidos. [WHO safe childbirth checklist implementation guide: improving the quality of facility-based delivery for mothers and newborns]. Genebra: Organização Mundial da Saúde, 2017.

ROSCANI, Alessandra Nazareth Cainé Pereira et al. Validação de checklist cirúrgico para prevenção de infecção de sítio cirúrgico. Acta paul. enferm. São Paulo, v.28, n.6, p.553565, dez. 2015. Disponível em: <http://www.scielo.br>. Acesso em: 03 nov., 2017.

WALDOW, Vera Regina. Cuidado colaborativo em instituições de saúde: a enfermeira como integradora. Texto \& Contexto Enfermagem, v.23, n.4, 2014. 